\title{
Article \\ A Subclass of Janowski Starlike Functions Involving Mathieu-Type Series
}

\author{
Dong Liu ${ }^{1}$, Serkan Araci ${ }^{2, *(1)}$ and Bilal Khan ${ }^{3}(\mathbb{D}$ \\ 1 Department of Information Technology, Luoyang Normal University, Louyang 471934, China; \\ liudong@lynu.edu.cn \\ 2 Department of Economics, Faculty of Economics Administrative and Social Sciences, \\ Hasan Kalyoncu University, Gaziantep TR-27410, Turkey \\ 3 School of Mathematical Sciences and Shanghai Key Laboratory of PMMP, East China Normal University, \\ 500 Dongchuan Road, Shanghai 200241, China; bilalmaths789@gmail.com \\ * Correspondence: serkan.araci@hku.edu.tr
}

check for updates

Citation: Liu, D.; Araci, S.; Khan, B. A Subclass of Janowski Starlike Functions Involving Mathieu-Type Series. Symmetry 2022, 14, 2. https:// doi.org/10.3390/sym14010002

Academic Editor: Clemente Cesarano

Received: 29 November 2021 Accepted: 16 December 2021 Published: 21 December 2021

Publisher's Note: MDPI stays neutral with regard to jurisdictional claims in published maps and institutional affiliations.

Copyright: (C) 2021 by the authors. Licensee MDPI, Basel, Switzerland. This article is an open access article distributed under the terms and conditions of the Creative Commons Attribution (CC BY) license (https:// creativecommons.org/licenses/by/ $4.0 /)$.

\begin{abstract}
To date, many interesting subclasses of analytic functions involving symmetrical points and other well celebrated domains have been investigated and studied. The aim of our present investigation is to make use of certain Janowski functions and a Mathieu-type series to define a new subclass of analytic (or invariant) functions. Our defined function class is symmetric under rotation. Some useful results like Fekete-Szegö functional, a number of sufficient conditions, radius problems, and results related to partial sums are derived.
\end{abstract}

Keywords: analytic functions; starlike function; Janowski function; Mathieu-type series

MSC: 30C45, 30C50

\section{Introduction and Motivation}

Let $\mathcal{H}$ represent the class of analytic function inside open unit disk

$$
\mathbb{D}=\{z: z \in \mathbb{C} \text { and }|z|<1\}
$$

and of the form

$$
f(z)=z+\sum_{n=2}^{\infty} a_{n} z^{n}, \quad(z \in \mathbb{D}) .
$$

Furthermore, for two given functions $g_{1}, g_{2} \in \mathcal{A}$, we say that $g_{1}$ is subordinate to $g_{2}$, (see for example $[1,2]$ ) and written symbolically as

$$
g_{1} \prec g_{2}
$$

if there exist a Schwarz function $\omega$, which is holomorphic in $\mathbb{D}$ with

$$
\omega(0)=0, \quad \text { and } \quad|\omega(z)|<1 \text {, }
$$

so that

$$
g_{1}(z)=g_{2}(\omega(z)), \quad(z \in \mathbb{D})
$$

Moreover, if the function $g_{2}$ is univalent in $\mathbb{D}$, then the following equivalence hold true:

$$
g_{1} \prec g_{2} \Longleftrightarrow g_{1}(0)=g_{2}(0) \text { and } g_{1}(\mathbb{D}) \subset g_{2}(\mathbb{D}) \text {. }
$$

For two analytic functions

$$
f(z)=\sum_{n=0}^{\infty} a_{n} z^{n}
$$


and

$$
g(z)=\sum_{n=0}^{\infty} b_{n} z^{n}, \quad(z \in E) .
$$

The convolution (Hadamard product) of $f(z)$ and $g(z)$ is defined as:

$$
f(z) * g(z)=\sum_{n=0}^{\infty} a_{n} b_{n} z^{n}
$$

Let we denote the class of Carathéodory functions by $\mathcal{P}$, for example a function $p$ is said to be in the class $\mathcal{P}$ if it has the following series form:

$$
p(z)=1+\sum_{n=1}^{\infty} c_{n} z^{n},
$$

such that

$$
\Re\{p(z)\}>0, \quad(\forall z \in \mathbb{D}) .
$$

Moreover, by class $\mathcal{S}$ we denote all functions of class $\mathcal{H}$ which are univalent inside open unit disc $\mathbb{D}$. Additionally, the class of starlike functions is denoted here by $\mathcal{S}^{*}$ and is defined as that a function $f \in \mathcal{H}$ is called starlike function if

$$
\Re\left(\frac{z f^{\prime}(z)}{f(z)}\right)>0, \quad(z \in \mathbb{D}),
$$

which equivalently, by using the principal of subordination can be written as:

$$
\mathcal{S}^{*}(\varphi)=\left\{f \in \mathcal{A}: \frac{z f^{\prime}(z)}{f(z)} \prec \varphi(z)\right\},
$$

where $\varphi(z)=(1+z) /(1-z)$. If we contrast the function $\varphi$ on the right hand side of (4), then we acquire several subclasses of $\mathcal{S}$ whose image domains have some exciting geometrical configurations as follows:

1. The functions class $\mathcal{S}^{*}(\varphi)$ with $\varphi(z)=1+\sin z$ was presented and studied by Cho et al. [3].

2. The functions class $\mathcal{S}^{*}(\varphi)$ with $\varphi(z)=1+z-\frac{1}{3} z^{3}$, maps the open unit disc onto the interior of the nephroid, a 2-cusped kidney-shaped region was familiarized and investigated by Wani and Swaminathan [4].

3. The functions class $\mathcal{S}^{*}(\varphi)$ with $\varphi(z)=1+\frac{4}{3} z+\frac{2}{3} z^{2}$ was presented by Sharma et al. [5].

4. The functions class $\mathcal{S}^{*}(\varphi)$ with $\varphi(z)=e^{z}$ was introduced and deliberated by Mendiratta et al. [6].

5. The functions class $\mathcal{S}^{*}(\varphi)$ with $\varphi(z)=z+\sqrt{1+z^{2}}$, which maps open unit disk to crescent shaped region, was given in [7].

For each of the above-defined functions classes many interesting properties were obtained, including some symmetrical and geometrical interpretation. In particular these classes posses a specific type of symmetry. Also, we note that lately various subclasses of starlike functions were introduced see [8-10], instead of the function $\varphi$ in (4), using some specific functions, such as those related with shell-like curves associated with Fibonacci numbers, Bell numbers, functions associated with conic domains and rational functions.

In the same way, if $\varphi(z)=\frac{1+A z}{1+B z}$ in (4) then, we have the following functions class.

Definition 1. A given function $f$ is said to belong to the class $\mathcal{S}^{*}[A, B]$ if and only if

$$
\frac{z f^{\prime}(z)}{f(z)} \prec \frac{1+A z}{1+B z}, \quad(-1 \leq B<A \leq 1) .
$$


or equivalently

$$
\frac{z f^{\prime}(z)}{f(z)}=\frac{(A+1) p(z)-(A-1)}{(B+1) p(z)-(B-1)}, \quad(-1 \leq B<A \leq 1) .
$$

The analytic functions class $\mathcal{S}^{*}[A, B]$ was introduced by Janowski [11]. Furthermore, this functions class has been generalized and studied by the many authors. For example, recently in [12], Hu et al. defined a new subclass of multivalent Janowski functions and found out some of its interesting properties. In particular, they made use of certain basic (or $q$-) calculus in order to define their class. Then, they gave certain interesting results, like coefficient bounds, radii of starlikeness and convexity, sufficiency criteria, growth theorem and distortion problem. In their paper published in Symmetry (see [12]), open some interesting step toward a more aggregate and comprehensive analysis of these functions. In our present work, we are essentially motivated by the work of $\mathrm{Hu}$ et al. [12]. In particular, we make use of certain Mathieu-type series and the Janowski functions in order to define our functions class. As far as we know, there is little work in the literature related to Mathieu-type series for the Janowski functions. The major purpose of this study is to begin an investigation into the properties of Mathieu-type series related to the Janowski functions. One may also attempt to apply this Mathieu-type series in order to generalize the works presented in [13-18].

The preceding series is named after Émile Leonard Mathieu (1835-1890), who explored it in his monograph [19] on the elasticity of solid bodies

$$
S(r)=\sum_{n=1}^{\infty} \frac{2 n}{\left(n^{2}+r^{2}\right)^{2}}, \quad(r>0) .
$$

The series $S(r)$ has a closed integral form provided by (see [20])

$$
S(r)=\frac{1}{r} \int_{0}^{\infty} \frac{t \sin (r t)}{e^{t}-1} d t
$$

The Mathieu-type series is described as follows (see [21])

$$
S(r ; z)=\sum_{n=1}^{\infty} \frac{2 n}{\left(n^{2}+r^{2}\right)^{2}} z^{n}, \quad(r>0, \quad|z|<1) .
$$

This series was defined specifically for functions of real variables, however it was redefined for complex variables by Bansal et al. [22]. Since $S(r ; z) \notin \mathcal{H}$ so using following normalization, we have

$$
\begin{aligned}
S(r ; z) & =\frac{\left(r^{2}+1\right)^{2}}{2} \sum_{n=1}^{\infty} \frac{2 n}{\left(n^{2}+r^{2}\right)^{2}} z^{n} \\
& =z+\sum_{n=2}^{\infty} \frac{n\left(r^{2}+1\right)^{2}}{\left(n^{2}+r^{2}\right)^{2}} z^{n}
\end{aligned}
$$

for some related works, we refer the reader to see [23-25].

In Geometric Function Theory, the study of operators is significant. Convolution of certain analytic functions may be used to express several differential and integral operators. This formalism allows for further mathematical research and assists in a better understanding of the geometric and symmetric aspects of such operators. The significance of convolution in operator theory may be explained via [26-29].

Using the Hadamard product in conjunction with (1) and (6), we introduced a new linear operator $Q(n, r): \mathcal{A} \rightarrow \mathcal{A}$ as follows 


$$
Q(n, r) f=f(z) * S(r ; z)=z+\sum_{n=2}^{\infty} \frac{n\left(r^{2}+1\right)^{2}}{\left(n^{2}+r^{2}\right)^{2}} a_{n} z^{n} .
$$

Motivated by a recently published article by Shi et al. [30], in which they have found estimates for some coefficient functionals for three leaf-type starlike functions, from [31] where coefficient bounds for certain subclasses of analytic functions connected with Faber polynomial have been derived, and some other related works on this subject (see for example [32-35]). We will now define the following concepts:

Definition 2. A given function $Q(n, r) f$ having form (7) be in the class $\mathcal{S}_{\mathcal{M}}^{*}(A, B)$ if and only if

$$
\frac{z(Q(n, r) f)^{\prime}(z)}{Q(n, r) f} \prec \frac{1+A z}{1+B z}, \quad(z \in \mathbb{D}),
$$

Or equivalently, we can write the above subordination as follow:

$$
\left|\frac{z(Q(n, r) f)^{\prime}(z)-Q(n, r) f(z)}{A Q(n, r) f(z)-B z(Q(n, r) f)^{\prime}(z)}\right|<1 .
$$

Many mathematicians have been focused on problems involving the coefficients of functions $f$ in a certain subclass of $\mathcal{A}$ since the early twentieth century. De Branges solved the most significant and inspirational problem, the Bieberbach hypothesis, only 70 years after it was formulated. Many intriguing problems involving these coefficients have emerged throughout the years. The Fekete-Szegö functional $\left|a_{3}-a_{2}^{2}\right|$ is now one of the most key results regarding the coefficients of functions $f$. The generalization of this functional is as $\left|a_{3}-\mu a_{2}^{2}\right|$ for some $\mu$ real as well as complex. Here in this article, we find the Fekete-Szegö functional $\left|a_{3}-\mu a_{2}^{2}\right|$ for our defined functions class $\mathcal{S}_{\mathcal{M}}^{*}(A, B)$. To find the Fekete-Szegö problem, the following Lemma is needed.

Lemma 1 ([36,37]). Let $p \in \mathcal{P}$ and of the form (3). Then, for any complex number $v$

$$
\left|c_{2}-v c_{1}^{2}\right| \leqq 2 \max \{1,|1-2 v|\} .
$$

Specifically, if the number $v$ is a real parameter, then

$$
\left|c_{2}-v c_{1}^{2}\right| \leqq \begin{cases}-4 v+2 & (v \leqq 0) \\ 2 & (0 \leqq v \leqq 1) \\ 4 v-2 & (v \geqq 1) .\end{cases}
$$

In this investigation, we define a new subclass of Janowski starlike functions which involves a Mathieu-type series. We then study some useful results like Fekete-Szegö functional, a number of sufficient conditions, radius problems, and results related to partial sums.

\section{Main Results}

Theorem 1. Let $Q(n, r) f \in \mathcal{S}_{\mathcal{M}}^{*}(A, B)$. Then for a complex number $\mu$,

$$
\left|a_{3}-\mu a_{2}^{2}\right| \leq \frac{(A-B)\left(r^{2}+9\right)^{2}}{6\left(r^{2}+1\right)^{2}} \max \left\{1,\left|\frac{\rho_{1}(A, B, n)-3 \mu(A-B)\left(r^{2}+4\right)^{4}}{2\left(r^{2}+9\right)^{2}\left(r^{2}+1\right)^{2}}\right|\right\},
$$

where

$$
\rho_{1}(A, B, n)=(4-2 A+4 B)\left(r^{2}+9\right)^{2}\left(r^{2}+1\right)^{2},
$$


Moreover, for a real $\mu$, we have the following

$$
\left|a_{3}-\mu a_{2}^{2}\right| \leq \begin{cases}\left(\frac{(A-B)\left(r^{2}+9\right)^{2}}{6\left(r^{2}+1\right)^{2}}\right)\left(\frac{\rho_{2}(A, B, n)-3 \mu(A-B)\left(r^{2}+4\right)^{4}}{\left(r^{2}+9\right)^{2}\left(r^{2}+1\right)^{2}}\right), & \left(\mu<\sigma_{1}\right), \\ \frac{(A-B)\left(r^{2}+9\right)^{2}}{3\left(r^{2}+1\right)^{2}}, & \left(\sigma_{1} \leq \mu \leq \sigma_{2}\right), \\ \left(\frac{(A-B)\left(r^{2}+9\right)^{2}}{6\left(r^{2}+1\right)^{2}}\right)\left(\frac{3 \mu(A-B)\left(r^{2}+4\right)^{4}-\rho_{2}(A, B, n)}{\left(r^{2}+9\right)^{2}\left(r^{2}+1\right)^{2}}\right), & \left(\mu>\sigma_{2}\right),\end{cases}
$$

where

$$
\begin{gathered}
\rho_{2}(A, B, n)=(2 A-2 B)\left(r^{2}+9\right)^{2}\left(r^{2}+1\right)^{2}, \\
\sigma_{1}=\frac{(2 A-4 B-2)\left(r^{2}+9\right)^{2}\left(r^{2}+1\right)^{2}}{3(A-B)\left(r^{2}+4\right)^{4}},
\end{gathered}
$$

and

$$
\sigma_{2}=\frac{(2 A-4 B+2)\left(r^{2}+9\right)^{2}\left(r^{2}+1\right)^{2}}{3(A-B)\left(r^{2}+4\right)^{4}}
$$

Proof. We start here by demonstrating that the inequalities (10) and (11) are true for $Q(n, r) f \in \mathcal{S}_{\mathcal{M}}^{*}(A, B)$. Since $Q(n, r) f \in \mathcal{S}_{\mathcal{M}}^{*}(A, B)$, therefore, we have the following subordination:

$$
\frac{z(Q(f ; S))^{\prime}(z)}{Q(f ; S)} \prec \frac{1+A z}{1+B z}
$$

The above subordination can also be written as:

$$
\frac{z(Q(n, r) f)^{\prime}(z)}{Q(n, r) f(z)}=\frac{(A+1) p(z)-(A-1)}{(B+1) p(z)-(B-1)}, \quad(-1 \leq B<A \leq 1) .
$$

Now, let us consider

$$
\begin{aligned}
\frac{(A+1) p(z)-(A-1)}{(B+1) p(z)-(B-1)} & =(A+1) p(z)-(A-1)[(B+1) p(z)-(B-1)]^{-1} \\
& =\frac{(A-1)}{(B-1)}\left[1-\left(\frac{A+1}{A-1}\right) p(z)\right]\left[1+\sum_{n=1}^{\infty}\left(\frac{(B+1)}{B-1} p(z)\right)^{n}\right] \\
& =\frac{(A-1)}{(B-1)}+\left(\frac{(A-1)(B+1)}{(B-1)^{2}}-\frac{(A+1)}{(B-1)}\right) p(z) \\
& +\left(\frac{(A-1)(B+1)^{2}}{(B-1)^{3}}-\frac{(A+1)(B+1)}{(B-1)^{2}}\right)(p(z))^{2}+\ldots
\end{aligned}
$$

If $p(z)=1+p_{1} z+p_{2} z^{2}+p_{3} z^{3}+\ldots$, then we have after some suitable simplification that

$$
\begin{aligned}
& \frac{(A+1) p(z)-(A-1)}{(B+1) p(z)-(B-1)} \\
& =\sum_{n=1}^{\infty} \frac{-2(B+1)^{n-1}}{(B-1)^{n}}+\left\{\sum_{n=1}^{\infty} \frac{2 n(A-B)(B+1)^{n-1}}{(B-1)^{n+1}} p_{1}\right\} z \\
& +\left\{\sum_{n=1}^{\infty} \frac{2 n(A-B)(B+1)^{n-1}}{(B-1)^{n+1}} p_{2}+\sum_{n=1}^{\infty} \frac{n(n+1)(A-B)(B+1)^{n}}{(B-1)^{n+2}} p_{1}^{2}\right\} z^{2} \ldots
\end{aligned}
$$


Now, we see that the series

$$
\sum_{n=1}^{\infty} \frac{-2(B+1)^{n-1}}{(B-1)^{n}}, \sum_{n=1}^{\infty} \frac{2 n(A-B)(B+1)^{n-1}}{(B-1)^{n+2}}, \sum_{n=1}^{\infty} \frac{n(n+1)(A-B)(B+1)^{n}}{(B-1)^{n+2}}
$$

converges to $1, \frac{1}{2}(A-B)$ and $-\frac{1}{4}(A-B)(B+1)$ respectively. Therefore, we have

$$
\begin{aligned}
\frac{(A+1) p(z)-(A-1)}{(B+1) p(z)-(B-1)} & =1+\frac{1}{2}(A-B) p_{1} z \\
& +\left\{\frac{1}{2}(A-B) p_{2}-\frac{1}{4}(A-B)(B+1) p_{1}^{2}\right\} z^{2}+\ldots
\end{aligned}
$$

Also,

$$
\frac{z(Q(n, r) f)^{\prime}(z)}{Q(n, r) f(z)}=1+\frac{2\left(r^{2}+1\right)^{2}}{3\left(r^{2}+4\right)^{2}} a_{2} z+\left\{\frac{6\left(r^{2}+1\right)^{2}}{\left(r^{2}+9\right)^{2}} a_{3}-\frac{4\left(r^{2}+1\right)^{4}}{\left(r^{2}+4\right)^{2}} a_{2}^{2}\right\} z^{2}+\ldots
$$

We find from the Equations (15) and (16) that

$$
a_{2}=\frac{(A-B)\left(r^{2}+4\right)^{2}}{4\left(r^{2}+1\right)^{2}} p_{1}
$$

and

$$
a_{3}=\frac{(A-B)\left(r^{2}+9\right)^{2}}{4\left(r^{2}+1\right)^{2}}\left[(A-2 B-1) p_{1}^{2}+2 p_{2}\right] \text {. }
$$

Thus, clearly, we find that

$$
\left|a_{3}-\mu a_{2}^{2}\right|=\frac{(A-B)\left(r^{2}+9\right)^{2}}{12\left(r^{2}+1\right)^{2}}\left|p_{2}-\zeta p_{1}^{2}\right|
$$

where

$$
\zeta=\frac{3 \mu(A-B)\left(r^{2}+4\right)^{2}}{4\left(r^{2}+4\right)^{2}\left(r^{2}+1\right)^{2}}-\frac{A-2 B-1}{2} .
$$

Finally, by using the Lemma 1 in connection with (19), the result asserted by Theorem 1 is obtained.

Theorem 2. Let $Q(n, r) f \in \mathcal{S}_{\mathcal{M}}^{*}(A, B)$. Then

$$
\sum_{n=2}^{\infty} \frac{n\left(r^{2}+1\right)^{2}}{\left(n^{2}+r^{2}\right)^{2}}(n-1+|B n-A|)\left|a_{n}\right| \leq A-B .
$$

Proof. Let $Q(n, r) f \in \mathcal{S}_{\mathcal{M}}^{*}(A, B)$. Then, (8) can be put in the form of Schwarz function $\omega(z)$ as

$$
\frac{z(Q(n, r) f)^{\prime}(z)}{Q(n, r) f(z)}=\frac{1+A \omega(z)}{1+B \omega(z)}, \quad(z \in \mathbb{D})
$$

Or equivalently

$$
\left|\frac{z(Q(n, r) f)^{\prime}(z)-Q(n, r) f(z)}{A Q(n, r) f(z)-B z(Q(n, r) f)^{\prime}(z)}\right|<1 .
$$


Consider

$$
\begin{aligned}
\left|\frac{z(Q(n, r) f)^{\prime}(z)-Q(n, r) f(z)}{A Q(n, r) f(z)-B z(Q(n, r) f)^{\prime}(z)}\right|= & \left|\frac{\sum_{n=2}^{\infty} \frac{n\left(r^{2}+1\right)^{2}}{\left(r^{2}+n^{2}\right)^{2}}[n-1] a_{n} z^{n}}{(A-B) z+\sum_{n=2}^{\infty} \frac{n\left(r^{2}+1\right)^{2}}{\left(r^{2}+n^{2}\right)^{2}}[B n-A] a_{n} z^{n}}\right| \\
& \leq \frac{\sum_{n=2}^{\infty} \frac{n\left(r^{2}+1\right)^{2}}{\left(r^{2}+n^{2}\right)^{2}}[n-1]\left|a_{n}\right|}{(A-B)-\sum_{n=2}^{\infty}\left(\frac{n\left(r^{2}+1\right)^{2}}{\left(r^{2}+n^{2}\right)^{2}}|B n-A|\right)\left|a_{n}\right|}<1,
\end{aligned}
$$

after simple computation we get the required inequality (37).

The following function define in (22), is an example for a function to be in the class $\mathcal{S}_{\mathcal{M}}^{*}(A, B)$.

Example 1. For the function

$$
f(z)=z+\sum_{n=2}^{\infty} \frac{\left(n^{2}+r^{2}\right)^{2}(A-B)}{n\left(r^{2}+1\right)^{2}(n-1+|B n-A|)} v_{n} z^{n}, \quad(z \in \mathbb{D}),
$$

such that $\sum_{n=2}^{\infty} v_{n}=1$, we have

$$
\begin{aligned}
& \sum_{n=2}^{\infty} \frac{n\left(r^{2}+1\right)^{2}}{\left(n^{2}+r^{2}\right)^{2}}(n-1+|B n-A|)\left|a_{n}\right| \\
& =\sum_{n=2}^{\infty} \frac{n\left(r^{2}+1\right)^{2}}{\left(n^{2}+r^{2}\right)^{2}}(n-1+|B n-A|)\left(\frac{\left(n^{2}+r^{2}\right)^{2}(A-B)}{n\left(r^{2}+1\right)^{2}(n-1+|B n-A|)} v_{n}\right) \\
& =(A-B) \sum_{n=2}^{\infty} v_{n}=(A-B) .
\end{aligned}
$$

Corollary 1. Let $Q(n, r) f \in \mathcal{S}_{\mathcal{M}}^{*}(A, B)$. Then

$$
\left|a_{n}\right| \leq \frac{\left(n^{2}+r^{2}\right)^{2}(A-B)}{n\left(r^{2}+1\right)^{2}(n-1+|B n-A|)}, \quad(n \geq 2) .
$$

Proof. The proof is simple and so therefore left to the readers.

Theorem 3. Let $Q(n, r) f \in \mathcal{S}_{\mathcal{M}}^{*}(A, B)$. Then

$$
r-\frac{\left(4+r^{2}\right)^{2}(A-B)}{2\left(r^{2}+1\right)^{2}(1+|2 B-A|)} r^{2} \leq|f(z)| \leq r+\frac{\left(4+r^{2}\right)^{2}(A-B)}{2\left(r^{2}+1\right)^{2}(1+|2 B-A|)} r^{2} .
$$

Proof. Consider

$$
\begin{aligned}
|f(z)| & =\left|z+\sum_{n=2}^{\infty} a_{n} z^{n}\right| \leq|z|+\sum_{n=2}^{\infty}\left|a_{n}\right||z|^{n} \\
& =r+\sum_{n=2}^{\infty}\left|a_{n}\right||r|^{n},
\end{aligned}
$$

since for $|z|=r<1$, we have $r^{n}<r^{2}$ for $n \geq 2$ and

$$
|f(z)| \leq r+r^{2} \sum_{n=2}^{\infty}\left|a_{n}\right|
$$


Comparably

$$
|f(z)| \geq r-r^{2} \sum_{n=2}^{\infty}\left|a_{n}\right|
$$

Now, from (37) implies that

$$
\sum_{n=2}^{\infty} \frac{n\left(r^{2}+1\right)^{2}}{\left(n^{2}+r^{2}\right)^{2}}(n-1+|B n-A|)\left|a_{n}\right| \leq A-B .
$$

But

$$
\begin{aligned}
& \sum_{n=2}^{\infty} \frac{2\left(r^{2}+1\right)^{2}}{\left(4+r^{2}\right)^{2}}(1+|2 B-A|)\left|a_{n}\right| \\
& \leq \sum_{n=2}^{\infty} \frac{n\left(r^{2}+1\right)^{2}}{\left(n^{2}+r^{2}\right)^{2}}(n-1+|B n-A|)\left|a_{n}\right| \leq A-B
\end{aligned}
$$

which gives

$$
\sum_{n=2}^{\infty}\left|a_{n}\right| \leq \frac{\left(4+r^{2}\right)^{2}(A-B)}{2\left(r^{2}+1\right)^{2}(1+|2 B-A|)}
$$

Theorem 4. Let $Q(n, r) f \in \mathcal{S}_{\mathcal{M}}^{*}(A, B)$. Then

$$
r-\frac{\left(4+r^{2}\right)^{2}(A-B)}{\left(r^{2}+1\right)^{2}(1+|2 B-A|)} r^{2} \leq\left|f^{\prime}(z)\right| \leq r+\frac{\left(4+r^{2}\right)^{2}(A-B)}{\left(r^{2}+1\right)^{2}(1+|2 B-A|)} r^{2}
$$

Proof. The proof is very similar to the proof of Theorem 3, thus it has been omitted.

Theorem 5. Let $f_{i} \in \mathcal{S}_{\mathcal{M}}^{*}(A, B)$ and have of the from

$$
f_{i}(z)=z+\sum_{n=2}^{\infty} a_{i, n} z^{n}, \quad(i=1,2,3, \ldots, k) .
$$

Then $H \in \mathcal{S}_{\mathcal{M}}^{*}(A, B)$, where

$$
H(z)=\sum_{i=1}^{k} c_{i} f_{i}(z) \text { with } \sum_{i=1}^{k}\left|c_{i}\right|=1
$$

Proof. From Theorem 2, we can write

$$
\sum_{n=2}^{\infty} \frac{n\left(r^{2}+1\right)^{2}}{\left(n^{2}+r^{2}\right)^{2}}(n-1+|B n-A|) a_{n} \leq A-B .
$$

Also,

$$
H(z)=\sum_{i=1}^{k} c_{i}\left(z+\sum_{n=2}^{\infty} a_{i, n} z^{n}\right)=z+\sum_{n=2}^{\infty}\left(\sum_{i=1}^{k} c_{i} a_{i, n}\right) z^{n}
$$


therefore

$$
\begin{aligned}
\sum_{n=2}^{\infty} \frac{n\left(r^{2}+1\right)^{2}}{\left(n^{2}+r^{2}\right)^{2}}(n-1+|B n-A|)\left|\sum_{i=1}^{k} c_{i} a_{i, n}\right| \\
=\sum_{i=1}^{k}\left[\sum_{n=2}^{\infty} \frac{n\left(r^{2}+1\right)^{2}}{\left(n^{2}+r^{2}\right)^{2}}(n-1+|B n-A|)\left|a_{i, n}\right|\right]\left|c_{i}\right| \\
\leq \sum_{i=1}^{k}(A-B)\left|c_{i}\right|=(A-B) \sum_{i=1}^{k}\left|c_{i}\right|=A-B,
\end{aligned}
$$

thus $H(z) \in \mathcal{S}_{\mathcal{M}}^{*}(A, B)$.

Theorem 6. Let $f_{i} \in \mathcal{S}_{\mathcal{M}}^{*}(A, B)$, for $i=1,2, \ldots, j$. Then the arithmetic mean $A$ of $f_{i}$ is given by

$$
A(z)=\frac{1}{j} \sum_{n=1}^{j} f_{i}(z)
$$

and also belong to class $\mathcal{S}_{\mathcal{M}}^{*}(A, B)$.

Proof. From (28), we can write

$$
\begin{aligned}
A(z) & =\frac{1}{j} \sum_{n=1}^{j} f_{i}(z)=\frac{1}{j} \sum_{n=1}^{j}\left(z+\sum_{n=2}^{\infty} a_{j, n} z^{n}\right) \\
& =z+\sum_{n=2}^{\infty}\left(\frac{1}{j} \sum_{n=1}^{j} a_{j, n}\right) z^{n}
\end{aligned}
$$

to show $A(z)$ belong to $\mathcal{S}_{\mathcal{M}}^{*}(A, B)$, it is enough to show that

$$
\sum_{n=2}^{\infty} \frac{n\left(r^{2}+1\right)^{2}}{\left(n^{2}+r^{2}\right)^{2}}(n-1+|B n-A|)\left|\frac{1}{j} \sum_{n=1}^{j} a_{j, n}\right| \leq A-B .
$$

Consider

$$
\begin{gathered}
\sum_{n=2}^{\infty} \frac{n\left(r^{2}+1\right)^{2}}{\left(n^{2}+r^{2}\right)^{2}}(n-1+|B n-A|)\left|\frac{1}{j} \sum_{n=1}^{j} a_{j, n}\right| \\
=\frac{1}{j} \sum_{n=1}^{j}\left(\sum_{n=2}^{\infty} \frac{n\left(r^{2}+1\right)^{2}}{\left(n^{2}+r^{2}\right)^{2}}(n-1+|B n-A|)\left|a_{j, n}\right|\right) \\
\leq \frac{1}{j} \sum_{n=1}^{j}(A-B)=(A-B),
\end{gathered}
$$

this show that $A(z)$ belong to $\mathcal{S}_{\mathcal{M}}^{*}(A, B)$.

Theorem 7. Let $Q(n, r) f \in \mathcal{S}_{\mathcal{M}}^{*}(A, B)$, then $f$ is starlike functions of order $\beta(0 \leq \beta<1)$ for $|z|<r^{*}$, where

$$
r^{*}=\left(\frac{(1-\beta) n\left(r^{2}+1\right)^{2}}{(n-\beta)\left(n^{2}+r^{2}\right)^{2}} \frac{(n-1+|B n-A|)}{A-B}\right)^{\frac{1}{n-1}} .
$$

Proof. Let $Q(n, r) f \in \mathcal{S}_{\mathcal{M}}^{*}(A, B)$. To prove $f$ is in class of starlike functions of order $\beta$, it's enough to show that

$$
\left|\frac{z f^{\prime}(z)-f(z)}{z f^{\prime}(z)+(1-2 \beta) f(z)}\right|<1
$$


Using basic simplifications, we arrive at

$$
\sum_{n=2}^{\infty}\left(\frac{n-\beta}{1-\beta}\right)\left|a_{n}\right||z|^{n-1}<1
$$

Since $Q(n, r) f \in \mathcal{S}_{\mathcal{M}}^{*}(A, B)$, from (20) we have

$$
\sum_{n=2}^{\infty} \frac{n\left(r^{2}+1\right)^{2}}{\left(n^{2}+r^{2}\right)^{2}} \frac{(n-1+|B n-A|)}{A-B}\left|a_{n}\right|<1 .
$$

Inequality (29) will holds true if the following hold true:

$$
\sum_{n=2}^{\infty}\left(\frac{n-\beta}{1-\beta}\right)\left|a_{n}\right||z|^{n-1}<\sum_{n=2}^{\infty} \frac{n\left(r^{2}+1\right)^{2}}{\left(n^{2}+r^{2}\right)^{2}} \frac{(n-1+|B n-A|)}{A-B}\left|a_{n}\right|,
$$

which implies that

$$
|z|^{n-1}<\left(\frac{(1-\beta) n\left(r^{2}+1\right)^{2}}{(n-\beta)\left(n^{2}+r^{2}\right)^{2}} \frac{(n-1+|B n-A|)}{A-B}\right),
$$

thus we get required result.

Theorem 8. Let $f_{1}(z)=z$ and

$$
f_{n}(z)=z-\frac{\left(n^{2}+r^{2}\right)^{2}(A-B)}{n\left(r^{2}+1\right)^{2}(n-1+|B n-A|)} z^{n}, \quad(z \in \mathbb{D}, \quad n \geq 2) .
$$

Then $Q(n, r) f \in \mathcal{S}_{\mathcal{M}}^{*}(A, B)$ if and only if $Q(n, r) f$ can be expressed in the form

$$
Q(n, r) f(z)=\sum_{n=1}^{\infty} \delta_{n} f_{n}(z), \quad\left(\delta_{n} \geq 0\right),
$$

and

$$
\sum_{n=1}^{\infty} \delta_{n}=1
$$

Proof. From (31), we can easily write

$$
Q(n, r) f(z)=\sum_{n=1}^{\infty} \delta_{n} f_{n}(z)=z+\sum_{n=2}^{\infty} \delta_{n} \frac{\left(n^{2}+r^{2}\right)^{2}(A-B)}{n\left(r^{2}+1\right)^{2}(n-1+|B n-A|)} z^{n},
$$

then, form Theorem 2, we can write

$$
\begin{aligned}
\sum_{n=2}^{\infty} & \frac{n\left(r^{2}+1\right)^{2}}{\left(n^{2}+r^{2}\right)^{2}}(n-1+|B n-A|) \frac{\left(n^{2}+r^{2}\right)^{2} \delta_{n}}{n\left(r^{2}+1\right)^{2}(n-1+|B n-A|)}=(A-B) \sum_{n=2}^{\infty} \delta_{n} \\
& =(A-B)\left(1-\delta_{1}\right) \leq A-B .
\end{aligned}
$$

Thus by Theorem 2, $Q(n, r) f \in \mathcal{S}_{\mathcal{M}}^{*}(A, B)$. Conversly, let $Q(n, r) f \in \mathcal{S}_{\mathcal{M}}^{*}(A, B)$. Since the Theorem 2, we have

$$
\left|a_{n}\right| \leq \frac{\left(n^{2}+r^{2}\right)^{2}(A-B)}{n\left(r^{2}+1\right)^{2}(n-1+|B n-A|)}, \quad(n \geq 2),
$$


we set

$$
\delta_{n}=\frac{n\left(r^{2}+1\right)^{2}(n-1+|B n-A|)}{\left(n^{2}+r^{2}\right)^{2}(A-B)}\left|a_{n}\right|,(n \geq 2),
$$

and

$$
\delta_{1}=1-\sum_{n=2}^{\infty} \delta_{n}
$$

so it follows that

$$
f(z)=\sum_{n=1}^{\infty} \delta_{n} f_{n}(z)
$$

Hence the proof is completed.

\section{Results Related to Partial Sum}

In this section, we will look at the ratio of a function of the form (1) to its sequence of partial sums

$$
f_{j}(z)=z+\sum_{n=2}^{j} a_{n} z^{n}
$$

when the coefficients of $f$ are small enough to fulfill the criteria (20). We will set sharp lower bounds for

$$
\Re\left(\frac{f(z)}{f_{j}(z)}\right), \Re\left(\frac{f_{j}(z)}{f(z)}\right), \Re\left(\frac{f^{\prime}(z)}{f_{j}^{\prime}(z)}\right) \text { and } \Re\left(\frac{f_{j}^{\prime}(z)}{f^{\prime}(z)}\right) .
$$

Theorem 9. If $f$ of the form (1) satisfies condition (20), then

$$
\Re\left(\frac{f(z)}{f_{j}(z)}\right) \geq 1-\frac{1}{\zeta_{j+1}}, \quad(\forall z \in \mathbb{D}),
$$

and

$$
\Re\left(\frac{f_{j}(z)}{f(z)}\right) \geq \frac{\zeta_{j+1}}{1+\zeta_{j+1}}, \quad(\forall z \in \mathbb{D}),
$$

where

$$
\zeta_{j}=\frac{n\left(r^{2}+1\right)^{2}(n-1+|B n-A|)}{\left(n^{2}+r^{2}\right)^{2}(A-B)} .
$$

Proof. To show the inequality (32), we set:

$$
\begin{aligned}
\zeta_{j+1}\left[\frac{f(z)}{f_{j}(z)}-\left(1-\frac{1}{\zeta_{j+1}}\right)\right] & =\frac{1+\sum_{n=2}^{j} a_{n} z^{n-1}+\zeta_{j+1} \sum_{n=j+1}^{\infty} a_{n} z^{n-1}}{1+\sum_{n=2}^{j} a_{n} z^{n-1}} \\
& =\frac{1+\psi_{1}(z)}{1+\psi_{2}(z)} .
\end{aligned}
$$

We now set:

$$
\frac{1+\psi_{1}(z)}{1+\psi_{2}(z)}=\frac{1+\omega(z)}{1-\omega(z)} .
$$

Then, after some appropriate simplification, we find that:

$$
\omega(z)=\frac{\psi_{1}(z)-\psi_{2}(z)}{2+\psi_{1}(z)+\psi_{2}(z)} .
$$


Thus, clearly, we find that:

$$
\omega(z)=\frac{\zeta_{j+1} \sum_{n=j+1}^{\infty} a_{n} z^{n-1}}{2+2 \sum_{n=2}^{j} a_{n} z^{n-1}+\zeta_{j+1} \sum_{n=j+1}^{\infty} a_{n} z^{n-1}} .
$$

We arrived at the following inequality by applying the trigonometric inequalities with $|z|<1$,

$$
|\omega(z)| \leq \frac{\zeta_{j+1} \sum_{n=j+1}^{\infty}\left|a_{n}\right|}{2-2 \sum_{n=2}^{j}\left|a_{n}\right|-\zeta_{j+1} \sum_{n=j+1}^{\infty}\left|a_{n}\right|} .
$$

We can now see that:

$$
|\omega(z)| \leq 1
$$

if and only if

$$
2 \zeta_{j+1} \sum_{n=j+1}^{\infty}\left|a_{n}\right| \leq 2-2 \sum_{n=2}^{j}\left|a_{n}\right|,
$$

which implies that:

$$
\sum_{n=2}^{j}\left|a_{n}\right|+\zeta_{j+1} \sum_{n=j+1}^{\infty}\left|a_{n}\right| \leq 1 .
$$

Finally, to emphasize the inequality (32), it is sufficient to demonstrate that the left hand side of the (35) is bounded above by the following sum:

$$
\sum_{n=2}^{\infty} \zeta_{n}\left|a_{n}\right|
$$

which is equivalent to

$$
\sum_{n=2}^{j}\left(\zeta_{n}-1\right)\left|a_{n}\right|+\sum_{n=j+1}^{\infty}\left(\zeta_{n}-\zeta_{j+1}\right)\left|a_{n}\right| \geq 0 .
$$

In lights of (36), thus it completes the proof of inequality in (32).

Moreover, to find the inequality (33), we set:

$$
\begin{aligned}
\left(1+\zeta_{j+1}\right)\left(\frac{f_{j}(z)}{f(z)}-\frac{\zeta_{j+1}}{1+\zeta_{j+1}}\right) & =\frac{1+\sum_{n=2}^{j} a_{n} z^{n-1}-\zeta_{j+1} \sum_{n=j+1}^{\infty} a_{n} z^{n-1}}{1+\sum_{n=2}^{\infty} a_{n} z^{n-1}} \\
& =\frac{1+\omega(z)}{1-\omega(z)},
\end{aligned}
$$

where

$$
|\omega(z)| \leq \frac{\left(1+\zeta_{j+1}\right) \sum_{n=j+1}^{\infty}\left|a_{n}\right|}{2-2 \sum_{n=2}^{j}\left|a_{n}\right|-\left(\zeta_{j+1}-1\right) \sum_{n=j+1}^{\infty}\left|a_{n}\right|} \leq 1
$$


The final inequality in (37) can also be written as:

$$
\sum_{n=2}^{j}\left|a_{n}\right|+\zeta_{j+1} \sum_{n=j+1}^{\infty}\left|a_{n}\right| \leq 1
$$

Finally, we can observe that the inequality (38) on the left hand side is bounded above by the following sum:

$$
\sum_{n=2}^{\infty} \zeta_{n}\left|a_{n}\right|
$$

and we have completed the assertion's (33) proof. Finally, the proof of our Theorem 9 is now completed.

Next, we look at derivatives-based ratios.

Theorem 10. If $f$ of the form (1) satisfies condition (20), then

$$
\Re\left(\frac{f^{\prime}(z)}{f_{j}^{\prime}(z)}\right) \geq 1-\frac{j+1}{\zeta_{j+1}} \quad(\forall z \in \mathbb{D})
$$

and

$$
\Re\left(\frac{f_{j}^{\prime}(z)}{f^{\prime}(z)}\right) \geq \frac{\zeta_{j+1}}{\zeta_{j+1}+j+1} \quad(\forall z \in \mathbb{D}),
$$

where $\zeta_{j}$ is given by $(34)$.

Proof. Since the proof of Theorem 10 is equivalent to that of Theorem 9, we skip the corresponding details here.

\section{Concluding Remarks and Observation}

We have successfully studied the uses of certain Mathieu-type series and defined a new convolution operator. We have then used our defined operator and studied a subclass of starlike functions associated with the Janowski functions. We have then derived some useful results like Fekete-Szegö functional, a number of sufficient conditions, radius problems and results related to partial sums for our defined functions class.

In concluding our current investigation, we would like to bring to the attention of interested readers to the possibility of studying fundamental or quantum (or q-) generalizations of the results which we have elaborated on in this paper. The interested readers may also attempt to developed these results with symmetrical points. Srivastava's recently published survey-cum-expository review study [28] see also [29] has impacted and driven this research area. However, as Srivastava (see p. 340 [28] and Section 5, pp. 1511-1512 [33]) have previously proved the $(\mathfrak{p}, \mathfrak{q})$-variations of the intended $\mathfrak{q}$-results because the forced-in parameter $\mathfrak{p}$ is insignificant, it will lead to being insubstantial.

Author Contributions: Conceptualization, D.L., S.A. and B.K.; methodology, D.L., S.A. and B.K.; validation, D.L., S.A. and B.K.; formal analysis, D.L., S.A. and B.K.; investigation, D.L., S.A. and B.K.; resources, D.L., S.A. and B.K.; data curation, D.L., S.A. and B.K.; writing-original draft preparation, D.L., S.A. and B.K.; writing-review and editing, D.L., S.A. and B.K. All authors have read and agreed to the published version of the manuscript.

Funding: This research received no external funding.

Institutional Review Board Statement: Not applicable.

Informed Consent Statement: Not applicable.

Data Availability Statement: Not applicable. 
Acknowledgments: The authors are grateful to the editor and the reviewers for their valuable comments and suggestions.

Conflicts of Interest: The authors declare that they have no conflict of interest.

\section{References}

1. Miller, S.S.; Mocanu, P.T. Differential subordination and univalent functions. Mich. Math. J. 1981, 28, 157-171. [CrossRef]

2. Miller, S.S.; Mocanu, P.T. Differential Subordinations Theory and Applications; CRC Press: Boca Raton, FL, USA, 2000.

3. Cho, N.E.; Kumar, S.; Kumar, V.; Ravichandran, V. Radius problems for starlike functions associated with the sine function. Bull. Iran. Math. Soc. 2019, 45, 213-232. [CrossRef]

4. Wani, L.A.; Swaminathan, A. Starlike and convex functions associated with a Nephroid domain. Bull. Malays. Math. Sci. Soc. 2021 44, 79-104. [CrossRef]

5. Sharma, K.; Jain, N.K.; Ravichandran, V. Starlike functions associated with cardioid. Afr. Math. 2016, 27, 923-939. [CrossRef]

6. Mendiratta, R.; Nagpal, S.; Ravichandran, V. On a subclass of strongly starlike functions associated exponential function. Bull. Malays. Math. Sci. Soc. 2015, 38, 365-386. [CrossRef]

7. Raina, R.K.; Sokól, J. On coefficient estimates for a certain class of starlike functions. Hacet. J. Math. Stat. 2015, 44, 1427-1433. [CrossRef]

8. Kanas, S.; Răducanu, D. Some classes of analytic functions related to conic domains. Math. Slovaca 2014, 64, 1183-1196. [CrossRef]

9. Dzoik, J.; Raina, R.K.; Sokół, J. On certain subclasses of starlike functions related to a shell-like curve connected with Fibonacci numbers. Math. Comput. Model. 2013, 57, 1203-1211. [CrossRef]

10. Cho, N.E.; Kumar, S.; Kumar, V.; Ravichandran, V.; Srivastava, H.M. Starlike functions related to the Bell numbers. Symmetry 2019, 11, 219. [CrossRef]

11. Janowski, W. Some extremal problem for certain families of analytic functions I. Ann. Pol. Math. 1973, 28, 298-326. [CrossRef]

12. Hu, Q.; Srivastava, H.M.; Ahmad, B.; Khan, N.; Khan, M.G.; Mashwani, W.K.; Khan, B. A subclass of multivalent Janowski type $q$-starlike functions and its consequences. Symmetry 2021, 13, 1275. [CrossRef]

13. Islam, S.; Khan, M.G.; Ahmad, B.; Arif, M.; Chinram, R. q-Extension of Starlike Functions Subordinated with a Trigonometric Sine Function. Mathematics 2020, 8, 1676. [CrossRef]

14. Shi, L.; Srivastava, H.M.; Khan, M.G.; Khan, N.; Ahmad, B.; Khan, B.; Mashwani, W.K. Certain Subclasses of Analytic Multivalent Functions Associated with Petal-Shape Domain. Axioms 2021, 10, 291. [CrossRef]

15. Ebadian, A.; Cho, N.E.; Adegani, E.A.; Yalçın, S. New Criteria for Meromorphic Starlikeness and Close-to-Convexity. Mathematics 2020, 8, 847. [CrossRef]

16. Naeem, M.; Hussain, S.; Mahmood, T.; Khan, S.; Darus, M. A New Subclass of Analytic Functions Defined by Using Salagean q-Differential Operator. Mathematics 2019, 7, 458. [CrossRef]

17. Liu, L.; Liu, J.-L. Properties of Certain Multivalent Analytic Functions Associated with the Lemniscate of Bernoulli. Axioms 2021, 10, 160. [CrossRef]

18. Soybaş, D.; Joshi, S.B.; Pawar, H. On a Certain Subclass of Analytic Functions Involving Integral Operator Defined by Polylogarithm Function. Mathematics 2019, 7, 66. [CrossRef]

19. Mathieu, E.L. Traité de Physique Mathematique. VI-VII: Theory del Elasticité des Corps Solides (Part 2); Gauthier-Villars: Paris, France, 1890.

20. Emersleben, O. Über die Reihe $\sum_{n=1}^{\infty} n\left(n^{2}+c^{2}\right)^{-2}$. Math. Ann. 1952, 125, 165-171. [CrossRef]

21. Tomovski, Z. New integral and series representations of the generalized Mathieu series. Appl. Anal. Discrete Math. 2008, 2, 205-212. [CrossRef]

22. Bansal, D.; Sokól, J. Geometric properties of Mathieu-type power series inside unit disk. J. Math. Ineq. 2019, 13, 911-918. [CrossRef]

23. Nunokawa, M.; Sokól, J. On an extension of Sakaguchi's result. J. Math. Ineq. 2015, 9, 683-697. [CrossRef]

24. Bansal, D.; Sokól, J. Univalency of starlikeness of Harwitz-Lerch Zeta function inside unit disk. J. Math. Ineq. 2017, 11, 863-871. [CrossRef]

25. Sokól, J.; Witowicz, P. On an application of Vietoris's inequality. J. Math. Ineq. 2016, 10, 829-836. [CrossRef]

26. Khan, B.; Srivastava, H.M.; Arjika, S.; Khan, S.; Khan, N.; Ahmad, Q.Z. A certain $q$-Ruscheweyh type derivative operator and its applications involving multivalent functions. Adv. Differ. Equ. 2021, 2021, 279. [CrossRef]

27. Srivastava, H.M.; Ahmad, Q.Z.; Khan, N.; Khan. N.; Khan, B. Hankel and Toeplitz determinants for a subclass of $q$-starlike functions associated with a general conic domain. Mathematics 2019, 7, 1-15. [CrossRef]

28. Srivastava, H.M. Operators of basic (or $q$-) calculus and fractional $q$-calculus and their applications in geometric function theory of complex analysis. Iran J. Sci. Technol. Trans. A Sci. 2021, 44, 327-344. [CrossRef]

29. Srivastava, H.M.; Wanas, A.K.; Srivastava, R. Applications of the $q$-Srivastava-Attiya operator involving a certain family of bi-univalent functions associated with the Horadam polynomials. Symmetry 2021, 13, 1230. [CrossRef]

30. Shi, L.; Khan, M.G.; Ahmad, B.; Mashwani, W.K.; Agarwal, P.; Momani, S. Certain coefficient estimates problems for three-leaf-type starlike functions. Fractal Fract. 2021, 5, 137. [CrossRef]

31. Attiya, A.A.; Lashin, A.M.; Ali, E.E.; Agarwal, P. Coefficient bounds for certain classes of analytic functions associated with Faber polynomial. Symmetry 2021, 13, 302. [CrossRef] 
32. Shi, L.; Ahmad, B.; Khan, N.; Khan, M.G.; Araci, S.; Mashwani, W.K.; Khan, B. Coefficient Estimates for a subclass of meromorphic multivalent $q$-Close-to-convex functions. Symmetry 2021, 13, 1840. [CrossRef]

33. Srivastava, H.M. Some parametric and argument variations of the operators of fractional calculus and related special functions and integral transformatioons. J. Nonlinear Convex Anal. 2021, 22, 1501-1520.

34. Khan, M.G.; Ahmad, B.; Mostafa, S.; Mashwani, W.K.; Arjika, S.; Khan, B. Pascu-type analytic functions by Using Mittag-Leffler functions in Janowski domain. Math. Probl. Eng. 2021, 2021, 1209871.

35. Khan, M.G.; Ahmad, B.; Khan, N.; Mashwani, W.K.; Arjika, S.; Khan, B.; Chinram, R. Applications of Mittag-Leffer type poisson distribution to a subclass of analytic functions involving conic-type regions. J. Funct. Spaces 2021, 2021, 4343163. [CrossRef]

36. Keogh, F.R.; Merkes, E.P. A coefficient inequality for certain classes of analytic functions. Proc. Am. Math. Soc. 1969, 20, 8-12. [CrossRef]

37. Ma, W.; Minda, D. A unified treatment of some special classes of univalent functions. In Proceedings of the Conference on Complex Analysis, Tianjin, China, 19-23 June 1992; Li, Z., Ren, F., Yang, L., Zhang, S., Eds.; International Press: Cambridge, MA, USA, 1994; pp. 157-169. 